\title{
Raciocínio clínico: percepções e práticas de estudantes de medicina
}

\author{
Clinical reasoning: perceptions and practices of medical students
}

\author{
Daniel Moreira Paes Landim ${ }^{1}$ (1) danielpaeslandim@gmail.com \\ José Luiz Moreno-Neto' ${ }^{1}$ (D) neto_bahia27@yahoo.com.br

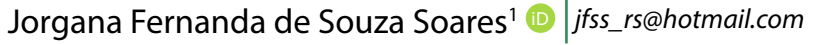

\section{RESUMO}

Introdução: Em tempos de ameaças constantes ao financiamento destinado à manutenção do Sistema Único de Saúde (SUS), faz-se necessário debruçar o olhar sobre a prática médica, sobretudo no que tange ao alto custo que o emprego desnecessário de exames complementares ocasiona à saúde pública.

Objetivo: Este estudo teve como objetivo analisar as percepções sobre o raciocínio clínico (RC) e as respectivas práticas entre estudantes de Medicina (EM) de uma universidade pública do Nordeste brasileiro.

Métodos: Trata-se de pesquisa qualitativa realizada com $12 \mathrm{EM}$. A coleta de dados ocorreu por meio de entrevistas semiestruturadas com gravação de áudio. A análise de dados foi realizada por meio da técnica de análise de conteúdo, modalidade análise temática.

Resultados: A maioria dos EM compreendeu o RC como sinônimo de raciocínio diagnóstico, pois os discentes o praticavam como uma junção da utilização de dados da anamnese, do exame físico e de exames complementares, e realizavam raciocínio terapêutico como base em aspectos como diagnóstico do paciente, sintomatologia, comorbidades, alergia e adesão terapêutica. Além disso, os EM demonstraram conhecimento teórico-conceitual superficial sobre o tema.

Conclusão: As percepções e práticas dos EM sobre o RC revelaram aspectos relevantes do contexto prático do internado na referida universidade, os quais podem subsidiar novas discussões e oportunizar a realização de outras pesquisas sobre o tema.

Palavras-chave: Educação Médica; Competência Clínica; Tomada de Decisão Clínica; Diagnóstico; Terapêutica.

\begin{abstract}
Introduction: In times of constant correction of funding for the maintenance of the Sistema Único de Saúde (SUS), it is necessary to focus on medical practice, especially with regard to the high cost that the unnecessary use of complementary tests causes to public health.

Objective: To analyze perceptions and practices in clinical reasoning (CR) of medical students at a public university in northeastern Brazil.

Methods: Qualitative research, conducted with 12 medical students (MS). Data was collected through semi-structured interviews with audio recording. Data analysis was performed using the method of thematic content analysis.

Results: Most MS understood clinical reasoning as a synonym for diagnostic reasoning, and CR practice as a combination of anamnesis data, physical exam and complementary exams. They perform therapeutic reasoning based on aspects such as patient diagnosis, symptoms, comorbidities, allergies and therapeutic compliance; and demonstrated superficial theoretical-conceptual knowledge of the topic.

Conclusion: The perceptions and practices of MS on CR revealed relevant aspects of the practical context of the internship at the university. The study, therefore, supports new discussions and opportunities for new research on the topic.
\end{abstract}

Keywords: Medical Education; Clinical Competence; Clinical Decision-Making. Diagnosis; Therapeutics.

${ }^{1}$ Faculdade de Medicina da Bahia - Universidade Federal da Bahia, Salvador, Bahia, Brasil.

Editora-chefe: Daniela Chiesa

Editor associado: Pedro Tadao Hamamoto Filho

Recebido em 15/06/20; Aceito em 04/09/20.

Avaliado pelo processo de double blind review. 


\section{INTRODUÇÃO}

Em tempos de ameaças constantes ao financiamento destinado à manutenção do Sistema Único de Saúde (SUS), faz-se necessário debruçar o olhar sobre a prática médica, sobretudo no que tange ao alto custo que o emprego desnecessário de exames complementares ocasiona à saúde pública. Nesse escopo, urge o direcionamento da atenção às tecnologias de baixo custo e comprovada eficiência para a resolução de problemas e manutenção da saúde da população brasileira de modo universal desde a formação médica, como é o caso do raciocínio clínico $(\mathrm{RC})$.

O RC é um elemento fundamental à prática médica em suas diferentes ações: diagnóstico, tratamento, rastreamento e outras. É conceituado como o caminho cognitivo percorrido pelo médico na tomada de decisões ${ }^{1}$, sendo a essência da perícia médica ${ }^{2}$. A importância do estudo do tema é comprovada quando se verifica a elevada proporção de erro diagnóstico ante mortem em autópsias e os registros de erros diagnósticos: $8 \%$ dos eventos adversos na profissão e até $30 \%$ das queixas de negligência. Por isso, o RC é uma habilidade decisiva para todos os médicos sem distinção ${ }^{3,4}$, independentemente de eles trabalharem em consultório particular ou em serviços de saúde em todos os níveis de complexidade do SUS.

Ao contrário do que se pode pensar, o RC não é uma habilidade de aplicação genérica para todos os problemas clínicos, mas sim um processo específico para cada contexto, com caráter multifatorial ${ }^{5}$. Atualmente, duas abordagens estão bem estabelecidas em sua execução: o raciocínio analítico e o não analítico. O primeiro utiliza abordagens hipotéticodedutivas e probabilísticas, estando amparado fortemente nas ciências biomédicas básicas. Já o segundo, por sua vez, emprega intuição, reconhecimento de padrões, roteiros de doenças (scripts) e heurística ${ }^{6}$. O raciocínio não analítico tem um caráter automático e se solidifica com a experiência, na resolução de problemas comuns, que possuem um nível de incerteza menor, e na escassez de tempo, com eficiência para situações rotineiras ${ }^{4}$, embora possa induzir a erros quando utilizado em casos de maior complexidade ${ }^{6}$. Nessas situações, o raciocínio analítico apresenta melhores resultados para responder a casos ambíguos e não rotineiros e quando existe maior disponibilidade de tempo 4 .

Os estudos atuais estabelecem como modelo mais aceito para o desenvolvimento do RC a teoria do processo dual que propõe a existência de uma integração entre o uso do raciocínio analítico e do não analítico ${ }^{4}$. Assim, transita-se entre essas duas formas de raciocínio a depender da experiência e da situação clínica em que o médico se encontre ${ }^{7}$. A pesquisa sobre RC é um campo em crescimento, mas carece de uma definição clara que seja aplicável tanto à prática clínica quanto à pesquisa, representando ainda um conceito vago, frequentemente expresso como sinônimo para outros objetos ${ }^{8}$. Essa lacuna no conhecimento, associada à necessidade de redução do quantitativo de erros diagnósticos, motivou o desenvolvimento deste estudo que objetivou analisar as percepções sobre o RC e as respectivas práticas entre estudantes de Medicina (EM) de uma universidade pública do Nordeste brasileiro.

\section{MÉTODOS}

Trata-se de uma pesquisa qualitativa. O método qualitativo é empregado em estudos de representações, da história, das relações, das crenças, das percepções e das opiniões, resultantes das interpretações que os indivíduos fazem sobre como vivem, pensam, sentem e constroem a si mesmos, como consequência da necessidade de captar algo do aspecto subjetivo da realidade social ${ }^{9}$. Logo, o foco de sua atenção busca mais a compreensão do que a explicação dos fenômenos ${ }^{10}$, possibilitando o desvelamento de processos sociais pouco conhecidos sobre grupos particulares, a revisão e criação de novos conceitos e categorias durante investigação, e a formulação de novas abordagens ${ }^{9}$.

Teve como cenário de estudo uma Faculdade de Medicina (FM) pública da Região Nordeste do Brasil. A matriz curricular do curso se estrutura a partir dos seguintes ciclos: básico, em que predominam as disciplinas das ciências biomédicas básicas; pré-clínico, no qual prevalece o estudo das especialidades médicas; e clínico ou internato, quando são colocados em prática os conhecimentos construídos ao longo do curso. Essa instituição possui cerca de mil alunos matriculados, dos quais 350 cursam o internato. Como critério de inclusão no estudo, o estudante deveria estar matriculado nas disciplinas Internato I ou II em Clínica Médica, oferecidas no quinto e sexto anos do curso. Excluíram-se aqueles que estavam afastados das atividades na FM por licença de saúde, licença-maternidade ou férias.

O critério empregado para o encerramento da coleta de dados na pesquisa ocorreu por saturação teórica, com a suspensão da inclusão de novos participantes a partir do momento em que ocorreu repetição de dados coletados ${ }^{11}$. Os EM foram entrevistados entre agosto e setembro de 2017. A coleta de dados ocorreu nas dependências da FM por meio de entrevistas semiestruturadas e gravadas, as quais foram realizadas em sala reservada a fim de não expor os participantes. Optou-se pela realização de entrevistas porque "entrevista é acima de tudo uma conversa a dois, ou entre vários interlocutores, realizada por iniciativa do investigador, destinada a construir informações pertinentes para um objetivo de pesquisa, e abordagem pelo entrevistador, de temas igualmente pertinentes tendo em vista este objetivo" (p. 261) ${ }^{9}$. 
Para as entrevistas, empregou-se um roteiro previamente elaborado com base na revisão de literatura e testado em estudo-piloto, que foi conduzido para analisar a sua adequação na elucidação do objetivo/objeto da pesquisa. Nesse roteiro, as questões versavam sobre os seguintes aspectos: características dos entrevistados (sexo e idade), entendimento sobre o RC e as formas utilizadas para elaborá-lo, maneiras de desenvolver o raciocínio diagnóstico e o terapêutico, e o conhecimento sobre como eles foram construídos ao longo do curso. Para este estudo, optou-se por apresentar somente os resultados referentes à compreensão acerca do que é e como se faz o $\mathrm{RC}$, bem como os seus elementos constitutivos - raciocínios diagnóstico e terapêutico. As entrevistas, posteriormente, foram transcritas na íntegra.

Para a análise dos dados obtidos, utilizou-se a técnica de análise de conteúdo, definida como um conjunto de estratégias analíticas da comunicação que visa à obtenção, por meio de procedimentos objetivos e sistemáticos de descrição do conteúdo das mensagens, de indicadores (quantitativos ou não) que possibilitam a inferência de conhecimentos relativos às condições de recepção/produção dessas mensagens ${ }^{12}$. Dentre as modalidades de análise de conteúdo, foi utilizada a análise temática, por ser considerada apropriada para as investigações qualitativas em saúde ${ }^{9}$ que buscam descobrir os núcleos de sentido que compuseram os dados coletados. A operacionalização da análise temática desdobrou-se nas seguintes etapas: pré-análise, exploração do material e tratamento dos resultados obtidos, e interpretação? As categorias empíricas de análise construídas foram: 1. percepções sobre RC e 2. prática do RC.

A execução da pesquisa foi aprovada pelo Comitê de Ética em Pesquisa da Faculdade de Medicina da Universidade Federal da Bahia (UFBA) em 11 de agosto de 2017 (Parecer nº 2.214.649). A realização das entrevistas foi autorizada mediante a assinatura do Termo de Consentimento Livre e Esclarecido em duas vias: uma do participante e outra do entrevistador. Nele, constava explicação pormenorizada sobre a natureza da pesquisa, os objetivos, métodos, benefícios previstos, potenciais riscos e incômodos. Para a garantia do anonimato, os estudantes foram identificados como EM, além do numeral arábico correspondente à ordem das entrevistas: 01 a 12.

\section{RESULTADOS}

Participaram do estudo 12 EM: nove homens e três mulheres, com idades entre 23 e 32 anos.

No que tange às percepções sobre o RC, a maior parte dos entrevistados compreendeu RC como sinônimo de raciocínio diagnóstico:
Raciocínio clínico eu acho que é o processo de raciocínio que você faz até chegar a um diagnóstico (EM 01).

Raciocínio clínico é o curso de pensamento para você chegar a diagnósticos (EM 02).

Também existiu a compreensão por parte de alguns estudantes de que o RC é uma junção da utilização de dados da anamnese, do exame físico e de exames complementares, com o objetivo de chegar a um diagnóstico e à terapêutica correspondente.

Eu acho que raciocínio clínico é a capacidade que você tem de com os dados que você tem do paciente, dos dados clínicos do paciente, com o histórico do paciente, você montar um possível diagnóstico, para a partir de então você tomar as condutas corretas para o paciente (EM 03).

É uma série de artifícios que o médico e o estudante lançam mão pra chegar a um diagnóstico e estabelecer uma terapêutica. Então, ele vai utilizar a anamnese, o exame físico e também os exames complementares, pra a partir daí chegar a um raciocínio com base no que ele colheu da história do que ele viu dos exames. $E$ a partir daí tentar chegar a um diagnóstico e instituir uma terapêutica (EM 06).

Quando questionados sobre o raciocínio terapêutico, os estudantes relataram a utilização de diferentes critérios, sendo o principal deles o uso da terapêutica com base no diagnóstico do paciente.

O raciocínio terapêutico a princípio depende, claro, né?, de você chegar a esse diagnóstico. A partir desse diagnóstico que você chega, você vai buscar com ajuda de treinamento, buscar em literaturas, em livros, o que é melhor para aquele paciente (EM 03).

Outros critérios empregados pelos estudantes no raciocínio terapêutico estão relacionados aos sinais e sintomas apresentados pelos pacientes, assim como às suas características pessoais, como: idade, sexo, comorbidades, alergias e adesão ao tratamento.

Então, primeiro a partir de algumas queixas a gente vai tentar. [...]. Como aqui é uma medicina alopata, a gente tenta diminuir o que o paciente está sentindo. Por exemplo, o paciente tem dor; dependendo da dor, a gente vai tentar diminuir; se a dor dele for um câncer, se a dor dele for infecção, se a dor dele for inflamatória, a gente vai tentar diminuir a dor (EM 02).

Você usa idade, sexo, se esse paciente tem comorbidades, interação medicamentosa, se ele tem alguma alergia (EM 12).

Quando questionados sobre como praticam o RC, percebeu-se, durante as entrevistas, que a maior parte dos 
estudantes não dispunha de conhecimento teórico-conceitual aprofundado sobre o assunto, especialmente a respeito das formas de RC que realizavam. No universo dos entrevistados, apenas dois relataram que utilizam o raciocínio analítico, enquanto os demais não souberam explicar qual tipo de raciocínio desenvolviam.

Normalmente, nessa etapa do curso, por ter pouca experiência, o raciocínio clínico é analítico (EM 02).

Acho que é mais analítico (EM 01).

A maior parte dos estudantes relatou que utiliza, em suas práticas, a associação entre anamnese, exame físico e exames complementares no desenvolvimento do RC:

Então, desde a anamnese, a partir da anamnese a gente já começa a reunir alguns dados, anamnese $e$ exame físico, aí então a gente traça as necessidades de exames complementares [...] (EM 10).

Essa prática subsidia tanto a realização do $\mathrm{RC}$ analítico como a do não analítico. Parte dos estudantes afirmou que elabora lista de problemas, diagnósticos sindrômicos, diagnósticos etiológicos e diagnósticos diferenciais.

Basicamente, com as informações que o paciente passou pra gente, geralmente a gente tenta construir aquele diagnóstico sindrômico pra daí a gente tentar partir para as etiologias e aí sim conseguir definir exatamente o que o paciente pode ter ou mais de uma suspeita diagnóstica (EM 07).

Alguns estudantes mencionaram que realizam o RC não analítico principalmente por meio do reconhecimento de padrões.

Muitas vezes eu uso o reconhecimento de padrões quando é possível, às vezes não é possível. Quando a gente aprende o raciocínio clínico, a etapa correspondente ao raciocínio sindrômico me dá uma certa margem para eu poder estabelecer esse tipo de norte pra você achar de evolução, de doença. Padrão de apresentação de doença, padrão de sinais e sintomas (EM 04).

Apenas um dos entrevistados relatou que utiliza tanto o raciocínio analítico quanto o não analítico, a depender da situação clínica apresentada, o que configura a prática do duplo processo ou processo dual, como está registrado no trecho a seguir:

Não é bem analítico na verdade, ele é por identificação de padrões. Então na verdade não existe um raciocínio, existe uma correlação com um quadro clínico semelhante a um anterior que eu vi ou que eu estudei e com essa semelhança acaba se levantando algumas suspeitas diagnósticas. Em casos difíceis é onde realmente ocorre uma análise em que eu vou olhando a história, o IS [interrogatório sistêmico], exames complementares, quais são os sistemas acometidos por essas três áreas e a partir daí então procurando doenças que possam explicar o acometimento dos sistemas. Então é em duas formas, tanto realmente analítica e tanto por padrões, que vi uma vez antes, vi de novo, eu acho que é isso. Esse é o raciocínio (EM 02).

\section{DISCUSSÃO}

A maior parte dos EM compreendeu o RC como sinônimo de raciocínio diagnóstico, realizado, principalmente, pela junção de dados da anamnese, do exame físico e de exames complementares. A prática do RC ocorreu pela associação do uso de anamnese, exame físico, exames complementares e, em alguns casos, lista de problemas, diagnósticos sindrômicos, etiológicos e diferenciais. O principal critério utilizado no raciocínio terapêutico foi o diagnóstico do paciente. $A$ maioria dos EM dispunha de conhecimento teórico-conceitual superficial sobre o assunto.

A compreensão dos estudantes do RC como raciocínio diagnóstico pode estar relacionado ao fato de esse termo ainda ser considerado um conceito ambíguo na medicina, carecendo de melhor detalhamento na sua definição ${ }^{8}$. A esse respeito, considera-se importante ressaltar que o raciocínio diagnóstico é apenas um dos componentes do RC, o qual também abarca o raciocínio terapêutico, além da resolução de problemas médicos, sendo, portanto, um processo cognitivo para o embasamento de decisões clínicas ${ }^{13}$. Destarte, o RC representa um dos principais instrumentos do processo de trabalho médico e, por ser uma tecnologia leve-dura, de natureza não palpável, necessita de maior aprofundamento teórico durante a graduação, a fim de que os médicos o compreendam em sua totalidade e não somente apenas como uma habilidade inerente à sua formação.

A compreensão dos estudantes do RC se dá por meio da coleta de dados clínicos e laboratoriais para a instituição de uma terapêutica específica permite pensar que no processo de resolução de problemas médicos é utilizada uma sequência de diferentes etapas, que incluem: coleta de dados, análise de informações, estabelecimento de possíveis diagnósticos diferenciais, entre outras. Dessa forma, é oportuno ressaltar que a anamnese é insubstituível para a formulação de hipóteses diagnósticas e a tomada de decisões, e os exames complementares não devem ser utilizados para suprir deficiências de semiotécnica, pois há a possibilidade de eles levarem a erro diagnóstico e escolhas inadequadas de procedimentos terapêuticos ${ }^{13,14}$. Dessa forma, esses procedimentos podem se tornar iatrogênicos. Destarte, devese buscar qualificar, na graduação médica, a habilidade dos estudantes em instituir o diagnóstico e a terapêutica com base na clínica apresentada, empregando métodos diagnósticos complementares somente na investigação de situações não 
bem elucidadas durante o encontro entre médico e paciente.

$\mathrm{Na}$ fala dos estudantes, o raciocínio diagnóstico está implícito. Ele se relaciona com o processo de testar hipóteses, que passam a orientar a investigação acerca de uma doença. Nesse contexto, a experiência clínica é um fator que favorece a formação de conjuntos semânticos de raciocínio mais diversificados, constituindo assim uma rede de ligações entre características clínicas e categorias diagnósticas. Dessa forma, um problema diagnóstico pode ser considerado fácil ou difícil a depender do conhecimento e da experiência do médico ${ }^{15}$. O RC também é permeado pela resolução de problemas médicos que possuem mecanismos de execução e de compreensão complexos. Contudo, propõem-se, para a realização do processo de solução de problemas médicos, ações que incluem: identificação de pistas, determinação de objetivos, proposição de ações, implementação de ações e avaliação de resultados. Esses componentes ocorrem de forma interligada e dinâmica ${ }^{16}$, de tal modo que, quando se pensa em $\mathrm{RC}$, um importante processo existente é o julgamento acompanhado pela experiência, possuindo relação de dependência do conhecimento tácito ${ }^{17}$. Isso permite pensar que a visão ainda incipiente dos graduandos acerca do objeto investigado no presente estudo, na qual reconhecem os elementos do RC sem nominá-los adequadamente, seja decorrente da pouca prática profissional, ou seja, o RC é uma habilidade aprimorada ao longo da vida.

O raciocínio terapêutico, na fala dos estudantes, esteve atrelado ao diagnóstico, o que é incontestável. Contudo, a tomada de decisão terapêutica envolve a formulação de planos de ação que objetivam a mudança situacional de um problema entre o momento atual e um momento futuro, proporcionando melhorias no quadro apresentado pela pessoa. Essa mudança pode representar a cura, o alívio de um sofrimento, a prevenção de complicações ou a redução de preocupações por parte do paciente ${ }^{18}$. Destarte, o diagnóstico, apesar de ser um passo fundamental ao processo de resolução de problemas médicos, não representa o objetivo final dele, que corresponde à solução do problema clínico.

Talvez a maior ênfase dada ao raciocínio diagnóstico deva-se ao fato de as pesquisas em RC terem se centrado, preponderantemente, no raciocínio diagnóstico, sendo reservado ao terapêutico um espaço ainda incipiente. Alguns aspectos estão relacionados a isso, como maior atenção dada à melhoria da precisão diagnóstica e a maior facilidade no que se refere ao estabelecimento de um rótulo diagnóstico, pois o raciocínio terapêutico se relaciona às diversas particularidades individuais e de adesão terapêutica ${ }^{19}$.

O fato de considerar, além do diagnóstico clínico, as características dos pacientes é indiscutível para que se tenha sucesso na terapêutica proposta pelo médico. O conceito de adesão, de forma geral, representa a utilização dos medicamentos ou de outros procedimentos prescritos em pelo menos $80 \%$ do seu total, considerando horários, tempo de tratamento e doses. A adesão terapêutica sofre a influência de fatores intrínsecos ao indivíduo, como a percepção sobre sua doença, aspectos psicológicos e comportamentais, além de fatores externos, relacionados a aspectos socioculturais e econômicos. É indiscutível a importância da adesão terapêutica, pois dela dependem o êxito da terapêutica proposta, a prevenção de uma doença, o controle de uma doença crônica e a cura de uma enfermidade ${ }^{20}$.

Além disso, a terapêutica utilizada em favor do paciente não deve ser baseada apenas na sua sintomatologia, mas sim na necessidade de saúde dele, vista de forma ampliada ${ }^{21}$. É dizer que a seleção do tratamento necessita ser dirigida à pessoa acometida, ou seja, deve-se empregar um plano terapêutico singular, no qual o diagnóstico propriamente dito deixa de ser central no processo de tomada de decisão sobre a conduta mais adequada a ser seguida.

Os resultados obtidos referentes à caracterização do tipo do RC praticado pelos participantes estão dentro do esperado para graduandos de Medicina. O estudo realizado por Roberti et al. ${ }^{6}$ teve como objetivo compreender como o RC se desenvolve entre estudantes de graduação em Medicina e revelou que a maior parte deles (93\%) desenvolvia, na fase pré-clínica, RC baseado nos conhecimentos das ciências biomédicas. Já o uso do RC não analítico, automático, passou a ganhar corpo ao longo do terceiro, quarto e quinto anos do curso, na proporção de $25 \%$, $24 \%$ e $78,5 \%$, respectivamente, estando diretamente relacionado ao maior incremento de atividades práticas. Tais resultados apontam para, além da necessidade de incrementaro embasamento teórico sobre a temática, aumentar as atividades ao longo do curso, nas quais os estudantes possam exercitar mais frequentemente o RC e identificar, no seu cotidiano, os elementos que o constituem e diferenciam como um processo.

A utilização de diagnósticos sindrômicos com posterior delimitação de diagnósticos diferenciais, chegando-se à definição de um diagnóstico etiológico, pressupõe, na prática do $\mathrm{RC}$, a realização de um raciocínio mais analítico do que não analítico, já que um processo cognitivo mais elaborado é necessário, para além do simples reconhecimento de padrões. A sua utilização pode facilitar a formulação da lista de hipóteses diagnósticas etiológicas. Dessa forma, o conjunto de diagnósticos sindrômicos de um paciente pode auxiliar na delimitação dos diagnósticos etiológicos mais prováveis para o caso e, assim, melhor direcionar a linha de investigação etiológica e a realização de uma anamnese com informações mais acuradas ${ }^{22}$. O diagnóstico diferencial consiste em analisar de forma comparativa, diferentes doenças que podem se 
manifestar de modo semelhante, buscando-se a eliminação gradativa daquelas com menor probabilidade de ocorrer. Assim, não se devem considerar todas as doenças possíveis, mas as que possuem maior probabilidade de ocorrer em um determinado caso, para chegar ao diagnóstico mais provável ou o diagnóstico de certeza ${ }^{14}$. Nesse contexto, salienta-se que a epidemiologia tem essencial participação na construção dos objetos da prática médica, e muito do que se observa em manuais didáticos de clínica, tanto no que diz respeito à definição de caso de uma determinada doença (sintomas, sinais e características laboratoriais) quanto no que concerne à adequação terapêutica, é validado por algum tipo de investigação epidemiológica ${ }^{23}$. Em muitas circunstâncias, a escuta qualificada do paciente, um adequado exame físico e o conhecimento acerca da situação de saúde da população local - os agravos à saúde e doenças mais incidentes/prevalentes na região de atuação do médico - são suficientes para embasar o RC sobre o caso apresentado, reduzindo, irrefutavelmente, o ônus do SUS decorrente da realização de exames complementares desnecessários e muitas vezes potencialmente iatrogênicos.

O reconhecimento de padrões representa a forma mais comum de prática do processo não analítico, intuitivo, e consiste, como já mencionado, em estabelecer, de forma inconsciente, ligações entre situações clínicas e padrões armazenados na memória de longo prazo, por meio da identificação automatizada e do tratamento de informações clínicas e contextuais. É marcado pela rapidez que permite aos médicos formular hipóteses diagnósticas, sendo utilizado pelos clínicos independentemente de seu grau de especialização. Ao contrário do que se pode pensar, estudos recentes sobre o tema mostram que o raciocínio não analítico, que inclui o reconhecimento de padrões, tem, quando usado de forma apropriada, propensão a erros semelhantes ao do raciocínio analítico ${ }^{24}$. O reconhecimento de padrões ocorre por meio de categorização, utilização de "roteiros de doença" ou "modelos mentais", intuição e heurística. Um padrão estabelece, assim, o que é normal e o que é uma variação do normal ${ }^{6}$. Contudo, o seu uso por parte de médicos com pouca prática profissional pode ter resultados insatisfatórios, uma vez que requer a repetição do quadro clínico em muitos $\operatorname{casos}^{25}$. Já o roteiro de doença é a estrutura cognitiva que possibilita ao clínico armazenar e ordenar as informações clínicas mais relevantes, aprimorandoas a cada caso clínico novo de uma mesma patologia. A estrutura desses roteiros pode ser constituída por fatores predisponentes e manifestações clínicas, por exemplo ${ }^{26}$.

No contexto das atividades clínicas, os estudantes, frequentemente, deparam com sinais e sintomas que passam a ser reconhecidos como padrões, agregadores de conhecimento limitado acerca de mecanismos de causa-efeito, mas que incorporam robusta quantidade de informações relativas à sintomatologia das doenças. $\mathrm{O}$ reconhecimento de padrões possibilita a geração de diagnóstico rapidamente para problemas rotineiros, contudo, ao defrontar-se com problemas de maior complexidade, nos quais não existe um padrão reconhecido, o estudante precisa voltar seu raciocínio para a abordagem analítica, embasada nas ciências básicas pelo potencial maior de ocorrência de erros ${ }^{6}$. Além disso, também devem ser consideradas, na prática do reconhecimento de padrões, circunstâncias que acarretam prejuízo a ele, como tornar um padrão rígido demasiadamente, focar um repertório limitado de padrões e tentar encaixar o paciente em um padrão desejado ignorando outras informações potencialmente importantes ao caso $^{27}$, abrindo-se a possibilidade para a solicitação de exames complementares excessivos, uma vez que a escuta do paciente é interrompida devido à necessidade de objetificar as doenças ${ }^{28}$. Entretanto, pensa-se que, antes de tudo, a medicina se faz por meio dos olhos e ouvidos do médico assistente, ou seja, está atrelada à capacidade de o médico escutar o que o paciente tem a dizer e estar atento também aos sinais apresentados.

Atualmente, nos estudos produzidos sobre o RC, existe a tendência de explicar a sua prática a partir da teoria do processo dual, que congrega o uso integrado e flexível das técnicas hipotético-dedutivas, raciocínio analítico, e de reconhecimento de padrões, raciocínio não analítico, em que uma prevalece sobre outra, o que dependerá da natureza das situações clínicas em questão ${ }^{29}$, já que esses dois tipos de raciocínio estão inter-relacionados.

A apresentação do paciente inicia o processo. Se os principaisrecursosdaapresentaçãoforemreconhecidos nesse estágio, o modo não analítico (sistema 1) será ativado automaticamente. Esta característica ou padrão inicial pode ser a apresentação visual da doença ou lesão, como condições dermatológicas, fraturas, distúrbios endócrinos, etc. ou combinação de tais sintomas ou achados (síndromes), roteiros de doenças, etc. Se a apresentação não for reconhecida ou se houver ambiguidades na apresentação, o modo de raciocínio analítico (sistema 2) será ativado. Neste sistema, os dados são sistematicamente examinados e logicamente decididos. Como o ciclo de identificação de pistas, interpretação de pistas, geração de hipóteses e testes de hipóteses estão em vigor neste sistema, é mais lento que o sistema 1 e requer mais recursos em termos de trabalho cognitivo ${ }^{8}$.

Além disso, os dois sistemas interagem sinergicamente de diferentes formas - o reconhecimento de padrões fornece aos médicos hipóteses de forma mais rápida, que em sequência são descartadas ou confirmadas pelo processo analítico. Ao mesmo tempo, o processamento repetitivo do raciocínio analítico leva, 
com frequência, ao reconhecimento de padrões do raciocínio não analítico ${ }^{8}$. Assim, pode-se defender que ambos são parte de um mesmo processo, portanto interdependentes e mais efetivos quando empregados juntos durante a prática do RC.

Em grande parte dos cursos de Medicina, ao longo do mundo, o RC é foco importante de desafios e esforços no âmbito do ensino, seja com a realização de cursos específicos sobre o tema ou por meio de estágios e sessões clínicas ${ }^{30}$, essas últimas tradicionalmente muito usadas, contudo de pouca eficácia ${ }^{31}$. Já a realização de cursos estruturados sobre o tema parece ter eficácia superior ${ }^{13}$. Destarte, seria interessante que o RC fosse apreendido pelo estudante não só como habilidade a ser desenvolvida por mimetismo, mas antes apresentado teoricamente, a fim de que haja a compreensão/familiarização acerca do principal instrumento de trabalho médico desde o primeiro semestre do curso de graduação. Para tal, talvez seja necessária a explicitação desse conteúdo nas Diretrizes Curriculares Nacionais para que todas as escolas médicas o trabalhem obrigatoriamente como teoria que subsidia a prática.

O presente estudo possui como limite principal a natureza dos dados - qualitativos, os quais não permitem que haja a generalização dos resultados. Outrossim, embora o critério de encerramento da coleta de dados tenha sido adotado adequadamente, apenas 12 vozes de estudantes do internato foram ouvidas e, talvez, representem (ou não) a realidade do local onde a pesquisa foi realizada. Assim, sugere-se que sejam realizados novos estudos qualitativos e quantitativos, os quais englobem maior número de estudantes desde os semestres iniciais do curso médico, assim como os docentes das disciplinas mais tradicionalmente voltadas ao ensino da prática clínica em distintos cenários do ensino médico que possam reiterar ou refutar os resultados aqui obtidos, assim como compreender como o RC como conhecimento e habilidade é construído ao longo dos cursos de graduação.

\section{CONCLUSÃO}

No presente estudo, pôde-se constatar que o RC vai além do raciocínio diagnóstico, abarcando também o raciocínio terapêutico e a tomada de decisões médicas, que de igual forma precisam ser bem compreendidos, qualificando assim a prática médica e reduzindo erros na profissão, potencialmente geradores de iatrogenias. Outrossim, representa uma possível abertura para se pensar, ainda na graduação, acerca da efetividade das ações médicas centradas na solicitação de exames complementares muitas vezes onerosos e desnecessários.

Evidenciou-se que os estudantes perceberam o RC como a junção entre dados da anamnese, do exame físico e dos exames complementares, sendo o embasamento para o raciocínio terapêutico o diagnóstico do paciente, além de sintomatologia, características pessoais e adesão terapêutica. $\mathrm{O}$ conhecimento teórico-conceitual sobre RC pelos estudantes também se mostrou incipiente, o que revela a necessidade de o discente, antes de aprender a raciocinar clinicamente, estudar de forma estruturada na graduação a ciência existente por trás dessa habilidade.

\section{CONTRIBUIÇÃO DOS AUTORES}

Daniel Moreira Paes Landim colaborou no planejamento do projeto, na coleta e análise de dados, e na redação do artigo. José Luiz Moreno-Neto colaborou na análise crítica do artigo. Jorgana Fernanda de Souza Soares orientou o planejamento do projeto, a análise de dados e a redação e análise crítica do artigo.

\section{CONFLITO DE INTERESSES}

Declaramos não haver conflito de interesses neste estudo.

\section{FINANCIAMENTO}

Declaramos que não houve financiamento neste estudo.

\section{REFERÊNCIAS}

1. Neves FF, Pazin-Filho A. Raciocínio clínico na sala de urgência. Medicina (Ribeirao Preto). 2008;41 (3):339-46 [acesso em 25 jul 2018]. Disponível em: http://www.revistas.usp.br/rmrp/article/view/279/280.

2. Wu B, Wang M, Grotzer TA, Liu J, Johnson JM. Visualizing complex processes using a cognitive-mapping tool to support the learning of clinical reasoning. BMC Med Educ. 2016;16(1) [acesso em 27 jun 2018]. Disponível em: https://www.ncbi.nlm.nih.gov/pmc/articles/ PMC4994325/pdf/12909_2016_Article_734.pdf.

3. Pinnock R, Young $L$, Spence F, Henning $M$, Hazell W. Can think aloud be used to teach and assess clinical reasoning in graduate medical education? J Grad Med Educ. 2015;7(3):334-7 [acesso em 7 jan 2019]. Disponível em: https://www.ncbi.nlm.nih.gov/pmc/articles/PMC4597940/pdf/i19498357-7-3-334.pdf.

4. Pennaforte T, Moussa A, Loye N, Charlin B, Audétat MC. Exploring a new simulation approach to improve clinical reasoning teaching and assessment: randomized trial protocol. JMIR Res Protoc. 2016;5(1):e26 [acesso em 26 fev 2019]. Disponível em: https://www.ncbi.nlm.nih.gov/ pmc/articles/PMC4776024/.

5. Findyartini A, Hawthorne L, Mccoll G, ChiavaroliN. How clinical reasoning is taught and learned: cultural perspectives from the University of Melbourne and Universitas Indonesia. BMC Med Educ. 2016;16(185) [acesso em 29 ju. 2019]. Disponível em: https://www.ncbi.nlm.nih.gov/ pmc/articles/PMC4957336/pdf/12909_2016_Article_709.pdf.

6. Roberti A, Roberti MRF, Pereira ERS, Porto CC, Costa NMSC. Development of clinical reasoning in an undergraduate medical program at a Brazilian university. Sao Paulo Med J. 2016;134(2):110-5 [acesso em 13 ago 2019]. Disponível em: http://www.scielo.br/pdf/spmj/v134n2/1516-3180spmj-_2015_00080108.pdf.

7. Boushehri E, Arabshahi KS, Monajemi A. Clinical reasoning assessment through medical expertise theories: past, present and future directions. Med J Islam Repub Iran. 2015;29(222) [acesso em 16 jun 2019]. Disponível em: https://www. ncbi.nlm.nih.gov/pmc/articles/PMC4606956/pdf/MJIRI-29-222.pdf.

8. Yazdani S, Abardeh $\mathrm{MH}$. Clinical reasoning in medicine: a concept analysis. J Med Educ. 2017;16 (3):154-62 [acesso em 15 abr 2019] Disponível em: https://www.researchgate.net/profile/Shahram_Yazdani3/ publication/323116352_Clinical_Reasoning_in_Medicine_A_Concept_ Analysis/links/5a80815aaca272a73769dda6/Clinical-Reasoning-inMedicine-A-Concept-Analysis.pdf. 
9. Minayo MCS. O desafio do conhecimento: pesquisa qualitativa em saúde. 10aed. São Paulo: Hucitec; 2007.

10. Nogueira-Martins MCF, Bógus CM. Considerações sobre a metodologia qualitativa como recurso para o estudo das ações de humanização em saúde. Saúde Soc. 2004;13(3):49-51 [acesso em 15 abr 2019]. Disponível em: http://www.scielo.br/pdf/sausoc/v13n3/06.pdf.

11. Fontanella BJB, Ricas J, Turato ER. Amostragem por saturação em pesquisas qualitativas em saúde: contribuições teóricas. CadSaude Publica. 2008;4(1):17-27 [acesso em 22 jun 2018]. Disponível em: https:// www.scielosp.org/pdf/csp/2008.v24n1/17-27/pt.

12. Bardin L. Análise de conteúdo. 3a ed. Lisboa: Edições70; 2004.

13. Harendza S, Krenz I, Klinge A, Wendt U, Janneck M. Implementation of a Clinical Reasoning Course in the Internal Medicine trimester of the final year of undergraduate medical training and its effect on students'case presentation and differential diagnostic skills. GMS J Med Educ. 2017;34(5):1-13 [acesso em12 maio 2019]. Disponível em: https://www. ncbi.nlm.nih.gov/pmc/articles/PMC5704605/pdf/JME-34-66.pdf.

14. Porto CC, Porto AL. Semiologia médica. 7aed. Rio de Janeiro: Guanabara Koogan;2015.

15. Elstein AS, Schwarz A. Clinical problem solving and diagnostic decision making: selective review of the cognitive literature. BMJ. 2002; 324: 729-32 [acesso em 12 mai 2019]. Disponível em: https://www.bmj.com/ content/324/7339/729.

16. Kiesewetter J, Ebersbach R, Görlitz A, Holzer M, Fischer MR, Schmidmaier R. Cognitive problem solving patterns of medical students correlate with success in diagnostic case solutions. PLoS ONE. 2013;8(8):1-8 [acesso em 20 jun 2019]. Disponível em: https://journals.plos.org/plosone/article/ file?id=10.1371/journal.pone.0071486\&type=printable.

17. Durning S, ArtinoJr AR, PangaroL, Van Der V CP, SchuwirthL.Context and clinical reasoning: understanding the perspective of the expert's voice. Med Educ. 2011;45(9):927-38. doi: 10.1111/j.1365-2923.2011.04053.x.

18. Réa-Neto A. Raciocínio clínico - o processo de decisão diagnóstica e terapêutica. Ver Assoc Med Bras. 1998:44(4):301-11 [acesso em 16 maio 2019]. Disponível em: http://www.scielo.br/pdf/ramb/v44n4/1898.pdf.

19. Mcbee $E$, Ratcliffe T, Picho K, Schuwirth L, ArtinoJr AR, Yepes-Rio AM, et al. Contextual factors and clinical reasoning: differences in diagnostic and therapeutic reasoning in board certified versus resident physicians. BMC Med Educ. 2017;17(1):1-8 [acesso em 5 jul 2019]. Disponível em: https:// www.ncbi.nlm.nih.gov/pmc/articles/PMC5688653/pdf/12909_2017_ Article_1041.pdf.

20. Leite SN, Vasconcellos MPC. Adesão à terapêutica medicamentosa: elementos para a discussão de conceitos e pressupostos adotados na literatura. Ciênc Saúde Colet. 2003;8(3):775-82 [acesso em 2 jul 2019]. Disponível em: http://www.scielo.br/scielo.php?pid=S1413$81232003000300011 \&$ script=sci_abstract\&tlng=pt.
21. 21. Camargo Jr KR. A biomedicina. Physis. 2005;15 (supl15):177-201 [acesso em 10 jul 2019]. Disponível em: www.scielo.br/pdf/physis/v15s0/ v15s0a09.pdf.

22. Biselli PJ, Atta JA. Diagnóstico sindrômico. Rev Med. 2005;84(3-4):95101 [acesso em 22 jul 2019]. Disponível em: http://www.revistas.usp.br/ revistadc/article/view/59250/62265.

23. de CamargoJrKR. (Ir)racionalidade médica: os paradoxos da clínica. Physis. 1992;2:(1):203-30 [acesso em 25 jul 2019]. Disponível em http://www.scielo.br/scielo.php?script=sci_abstract\&pid=S010373311992000100008\&lng=pt\&tlng=pt.

24. PelacciaT, Tardif J, Triby E, Charlin B. An analysis of clinical reasoning through a recent and comprehensive approach: the dual-process theory. Med Educ. 2011; 16. doi: 10.3402/meo.v16i0.5890.

25. Lopes DM, Bregagnollo GH, Barbosa BM, Stamm ANF. The process of clinical reasoning among medical students. Rev Bras Educ Med. 2018;42(3):194-200 [acesso em 12 fev 2020]. Disponível em: http://www. scielo.br/pdf/rbem/v42n3/1981-5271-rbem-42-3-0194.pdf.

26. Montaldo LG, Herskovic LP. Teaching of clinical reasoning to medical students using prototypical clinical cases. Rev Med Chile. 2013;141(7):82330 [acesso em 23 jul 2019]. Disponível em:https://scielo.conicyt.cl/scielo. php?script=sci_arttext\&pid=S0034-98872013000700001\&lng=en\&nrm= iso\&tlng=en.

27. Freiwald $T$, Salimi $M$, Khaljani $E$, Harendza S. Pattern recognition as a concept for multiple-choice questions in a National Licensing Exam. BMC Med Educ. 2014;14 (232) [acesso em 15 jul 2019]. Disponível em: https:// www.ncbi.nlm.nih.gov/pmc/articles/PMC4289202/

28. Tesser CD. Cuidado clínico e sobre medicalização na atenção primária à saúde. Trab Educ Saúde. 2019;17(2):0020537 [acesso em 12 fev 2020]. Disponível em: http://www.scielo.br/scielo.php?script=sci arttext\&pid=S1981-77462019000200400\&lng=en\&nrm=iso.

29. NanthaYS. Probability or reasoning: current thinking and realistic strategies for improved medical decisions. Korean J Fam Med. 2017:38(6):315-21 [acesso em 26 jul 2019]. Disponível em: https://www.kjfm.or.kr/journal/ view.php?number $=2068$.

30. Gruppen LD. Clinical reasoning: defining it, teaching it, assessing it, studying it. West J Emerg Med. 2017;18(1):4-7 [acesso em 25 jul 2019]. Disponível em: https://www.ncbi.nlm.nih.gov/pmc/articles/PMC5226761/ pdf/wjem-18-4.pdf.

31. Al-Rumayyan A, Ahmed N, Al-Subait R, Al-Ghamdi G, Mahzari MM Mohamed TA, et al. Teaching clinical reasoning through hypotheticodeduction is (slightly) better than self-explanation in tutorial groups: an experimental study. Perspect Med Educ. 2018;7(2):93-9.doi: 10.1007/ s40037-018-0409-x. 